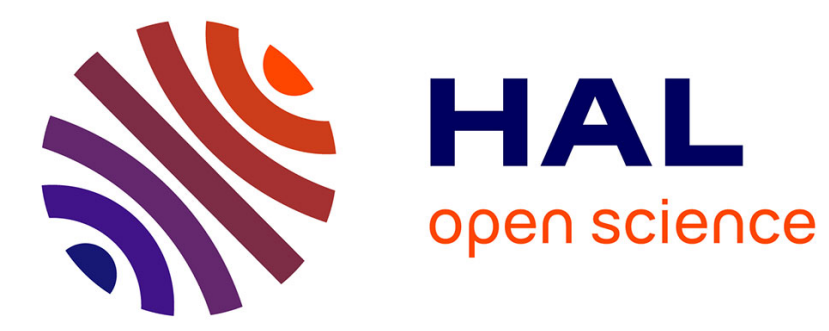

\title{
The Nonhomologous Nature of Solar Diameter Variations
}

Sabatino Sofia, Sarbani Basu, Pierre Demarque, Linghuai Li, Gérard Thuillier

\section{To cite this version:}

Sabatino Sofia, Sarbani Basu, Pierre Demarque, Linghuai Li, Gérard Thuillier. The Nonhomologous Nature of Solar Diameter Variations. The Astrophysical Journal, 2005, 432, pp.L147-L150. 10.1086/498068 . hal-00078977

\section{HAL Id: hal-00078977 \\ https://hal.science/hal-00078977}

Submitted on 19 Jul 2020

HAL is a multi-disciplinary open access archive for the deposit and dissemination of scientific research documents, whether they are published or not. The documents may come from teaching and research institutions in France or abroad, or from public or private research centers.
L'archive ouverte pluridisciplinaire HAL, est destinée au dépôt et à la diffusion de documents scientifiques de niveau recherche, publiés ou non, émanant des établissements d'enseignement et de recherche français ou étrangers, des laboratoires publics ou privés. 
The Astrophysical Journal, 632:L147-L150, 2005 October 20

(C) 2005. The American Astronomical Society. All rights reserved. Printed in U.S.A.

\title{
THE NONHOMOLOGOUS NATURE OF SOLAR DIAMETER VARIATIONS
}

\author{
Sabatino Sofia, ${ }^{1}$ Sarbani Basu, ${ }^{1}$ Pierre Demarque, ${ }^{1}$ Linghuai Li, ${ }^{1}$ and Gerard Thuillier ${ }^{2}$ \\ Received 2005 August 10; accepted 2005 September 14; published 2005 October 4
}

\begin{abstract}
We show in this Letter that the changes of the solar diameter in response to variations of large-scale magnetic fields and turbulence are not homologous. For the best current model, the variation at the photospheric level is over 1000 times larger than the variation at a depth of $5 \mathrm{Mm}$, which is about the level at which $f$-mode solar oscillations determine diameter variations. This model is supported by observations that indicate larger diameter changes for high-degree $f$-modes than for low-degree $f$-modes, since the energy of the former is concentrated at shallower layers than the latter.
\end{abstract}

Subject headings: Sun: fundamental parameters — Sun: helioseismology — Sun: magnetic fields

\section{INTRODUCTION}

The question of whether the solar diameter changes on timescales of years to centuries is very controversial. A recent paper (Thuillier et al. 2005b) presents a detailed summary of the issue. In essence, different measurement and analysis techniques, and sometimes even identical instruments and similar analysis methods, yield incompatible results. It is not presumptuous to infer that the cause of this controversy is that for the majority of the techniques, the results are at the borderline of the sensitivity of the technique.

The major exception to the above statement is the technique of helioseismology, particularly the $f$-modes of oscillation. Schou et al. (1997) and Antia (1998) have demonstrated that the frequencies of $f$-modes can be used to estimate the solar radius. Since these frequencies have been measured with a precision of one part in $10^{5}$, one can expect to determine the solar radius to similar precision. Changes in the $f$-mode frequencies have been used to determine changes in the solar radius (see, e.g., Dziembowski et al. 1998, Antia et al. 2000, etc.). The radius changes are estimated assuming that the fractional change in radius is uniform in the range of sensitivity of the method. The radius change determined by $f$-modes is the change at the radius where the $f$-modes are concentrated. One way of quantifying the depth at which the $f$-modes are sensitive is to look at the depth at which the energy of the $f$ modes is concentrated. This is shown in Figure 1, where we plot the density for $f$-modes of several degrees. The range of degrees on the plot reflects the range of available $f$-mode frequencies. We see that for the lowest degree mode in the figure $(l=140$ ), the peak of the energy is at about $6.3 \mathrm{Mm}$ (where temperature $T$ is about $41,000 \mathrm{~K}$ ), and for the highest degree $(l=300)$ it is at $3.3 \mathrm{Mm}(T=24,000 \mathrm{~K})$. There are, of course, other criteria by which one can determine radius (see, e.g., Cox 1980; Unno et al. 1989). However, here we show the energy density because that is the criterion used by various authors when discussing their radius-change results. In any event, the different methods do not significantly alter the conclusions of this Letter. It should be noted that although the $f$-modes have a precision of one part in $10^{5}$ or so, the changes in radius cannot be determined with this precision since that depends on

\footnotetext{
${ }^{1}$ Department of Astronomy, Yale University, P.O. Box 208101, New Haven, CT 06520-8101; sofia@astro.yale.edu, basu@astro.yale.edu, demarque@astro.yale.edu, li@astro.yale.edu.

${ }^{2}$ Service d'Aéronomie du CNRS, Bp 3, 91371 Verrières-le Buisson, France; gerard.thuillier@aerov.jussieu.fr.
}

how large the changes in $f$-modes are, and those changes happen to be very small. As a result, the radius-change measurements are not always very precise, as can be seen from the results shown below.

Consequently, even in the case of radius determination using $f$-mode oscillations, there does not seem to be consensus yet as to the exact amount by which the radius changes. The results obtained so far (Dziembowski et al. 1998, 2000, 2001; Antia et al. 2000, 2001; Antia \& Basu 2004) are not in agreement with each other. Dziembowski et al. (1998), using Michelson Doppler Imager (MDI) data, found that the solar radius reached a minimum around the minimum activity period in 1996 and was larger, by about $5 \mathrm{~km}, 6$ months before and after the minimum. However, later results, using longer time intervals, did not find any systematic changes (Dziembowski et al. 2000). On the other hand, Antia et al. (2000) using Global Oscillation Network Group (GONG) data found that the solar radius decreased by about $5 \mathrm{~km}$ between 1995 and 1998, and this variation appeared to be correlated (but in antiphase) with the level of solar activity. Subsequently, Antia et al. (2001), using both GONG and MDI data, put an upper limit of $1 \mathrm{~km} \mathrm{yr}^{-1}$ for the change in solar radius. Meanwhile, in a related paper, Dziembowski et al. (2001) claimed a solar radius decrease as a rate of $1.5 \mathrm{~km} \mathrm{yr}^{-1}$ during 1996-2000. Antia et al. (2001) made some sense of all these discrepant results by showing that the variation in $f$-mode frequencies could be divided into at least two components: one oscillatory, with a period of $1 \mathrm{yr}$, and a second, nonoscillatory, and probably correlated with solar activity. They argued that the oscillatory component is most likely an artifact introduced by the orbital period of the Earth. They also showed that most of the discrepancy between different results could be explained by using data sets that cover different time periods and by the failure to remove the oscillatory component. Upon performing those corrections, all the different investigations appear to indicate that the solar radius decreases with increasing solar activity.

In a more recent investigation Antia \& Basu (2004) examined the changes in $f$-mode frequencies using 8 years of MDI data. They obtained an upper limit of about $1 \mathrm{~km} \mathrm{yr}^{-1}$ for radius changes during the entire solar cycle. It is to be noted, however, that even this result is not very clear-cut, since different degree ranges of $f$-modes implied different radius changes. When the available higher degree modes were used $(140<l \leq 300)$, they got an average change of $-0.91 \pm 0.03 \mathrm{~km} \mathrm{yr}^{-1}$ between 1996 and 2004. The $f$-modes in the range $140 \leq l \leq 250$ show a change of $-0.41 \pm 0.04 \mathrm{~km} \mathrm{yr}^{-1}$ for the same period, but for 




Fig. 1.-Kinetic energy density of different $f$-modes. The curves are normalized by the total kinetic energy density in each mode. The eigenfunction used to calculate the energy density were those of the standard solar model of Basu et al. (2000).

$l<140$, no observable change was obtained $(\Delta R=0.13 \pm$ $0.20 \mathrm{~km} \mathrm{yr}^{-1}$ ). Antia \& Basu (2004) suggested that the difference in the results yielded by the different degree ranges indicated that the evidence for radius change was not conclusive. In this Letter we present an alternative interpretation of these observations, i.e., that the Sun does not expand or contract homologously with a change in solar activity.

\section{MODEL CALCULATIONS}

We construct models to calculate the change in solar radius with change in solar activity. The numerical code that we use to compute the structure and evolution of the solar model is an outgrowth of the Yale Rotating Evolution Code (YREC; Winnick et al. 2002) into which the effects of magnetic fields and turbulence have been included. The starting values of the basic solar parameters are $R_{\odot}=6.9598 \times 10^{10} \mathrm{~cm}$ and $L_{\odot}=3.8515 \times 10^{33} \mathrm{ergs} \mathrm{s}^{-1}$. These particular choices have negligible effects on the results. The version of the code used in these calculations is one-dimensional. The inclusion of magnetic fields considers their contribution to pressure and internal energy and their modification of energy transfer, primarily convection. The dynamical effects modify turbulent pressure and energy transport. The detailed formulation of the modifications to YREC is based on the approach first presented by Lydon \& Sofia (1995) and subsequently expanded by Li \& Sofia (2001) and $\mathrm{Li}$ et al. (2002, 2003).

Because the location, magnitude, and temporal behavior of the internal field are not known, we made two general assumptions: (1) the magnitude of the magnetic field would be that required to cause a luminosity change of $0.1 \%$ over the cycle and (2) the temporal behavior assumed is sinusoidal, and it mimics the shape of the activity cycle determined, for example, by the averaged sunspot number. We computed four cases (listed in Table 1), three with only magnetic fields at different depths, and one with both magnetic fields and turbulence. For the cases with only magnetic fields, the field con-
TABLE 1

Solar VARIABILITy Models

\begin{tabular}{lcllr}
\hline \hline Model & $\begin{array}{c}\text { Depth } \\
(\mathrm{Mm})\end{array}$ & $\begin{array}{c}\Delta B \\
(\mathrm{kG})\end{array}$ & $\begin{array}{c}P_{m} / P \\
(\%)\end{array}$ & $\begin{array}{r}P_{t} / P \\
(\%)\end{array}$ \\
\hline $1 \ldots \ldots$ & 4.45 & 4.2 & 0.66 & 0 \\
$2 \ldots \ldots$ & 2.43 & 1.7 & 1.1 & 0 \\
$3 \ldots \ldots$ & 0.38 & 0.15 & 0.67 & 0 \\
$4 \ldots \ldots$ & 2.33 & 0.38 & 1.2 & 16 \\
\hline
\end{tabular}

figuration was Gaussian. Guided by the observation of $p$-mode oscillations, we were led to the inclusion of turbulence ( $\mathrm{Li}$ et al. 2003). In this case, the properties of the turbulence were derived from numerical simulations of the outer region of the solar convective envelope (Robinson et al. 2003), and the magnetic field distribution was dictated by a feedback process between turbulence and magnetic field.

Although the specific details of the calculations reported here are contained in Li et al. (2003), we present in this Letter the results of the calculations that are relevant to the radius problem and not contained in that paper. The bottom panel of Figure 2 shows the difference in magnetic energy per unit mass $\left(\chi_{m} \equiv B^{2} / 8 \pi \rho\right)$ between the years 2000 and 1996 for all cases where only magnetic fields are taken into account. The top panel of the figure shows the ratio between the radius change as a function of radius to the radius change at $5 \mathrm{Mm}$.

Figure 3 is similar to Figure 2, but for the case in which turbulence (modulated by the magnetic field) is included. The bottom panel shows the difference in turbulent $\left[\chi_{t} \equiv \frac{1}{2}\left(v^{\prime \prime}\right)^{2}\right.$, where $v^{\prime \prime}$ is the magnitude of the turbulent velocity] plus magnetic $\left(\chi_{m}\right)$ energy per unit mass between the years 2000 and $1996\left(\chi=\chi_{m}+\chi_{t}\right)$, and the top panel shows the ratio between

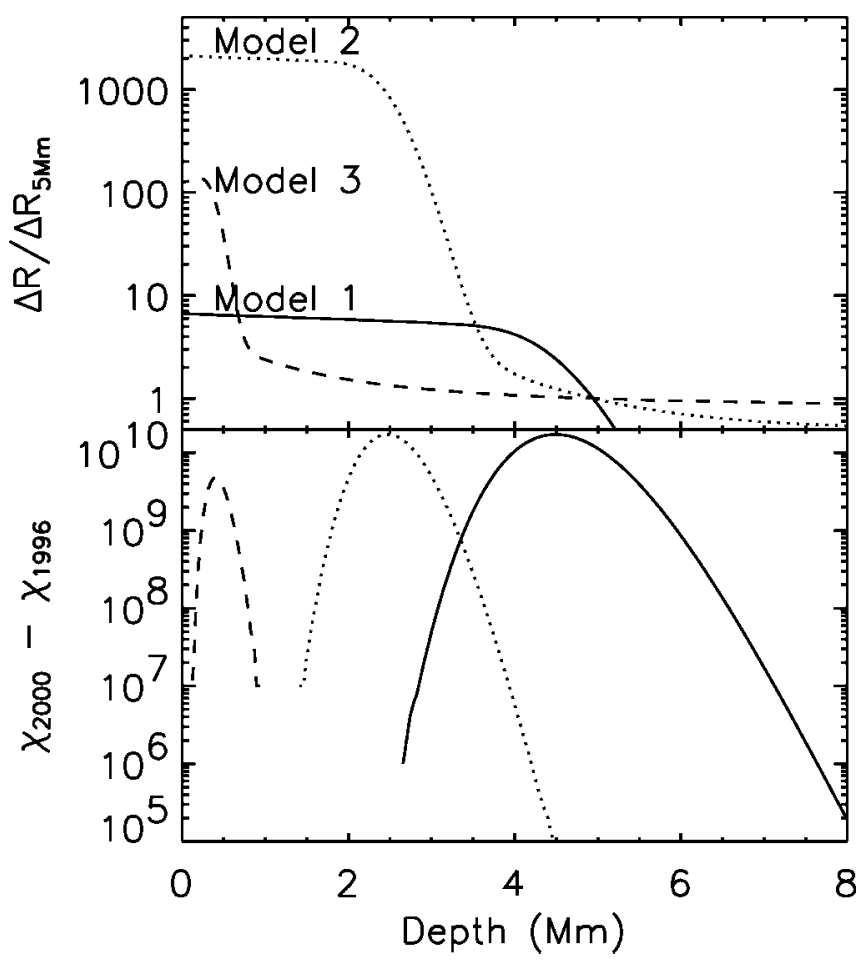

FIG. 2.-Bottom: Magnetic energy density for models 1 (solid line), 2 (dotted line), and 3 (dashed line). The model properties are listed in Table 1. Top: The ratio between radius change as a function of depth below the photosphere and radius change at $5 \mathrm{Mm}$ for models 1-3. 




FIG. 3.-Bottom: Magnetic plus turbulent energy density for model 4, where turbulence is modulated by the magnetic field. Note that the increase in magnetic field density between 1996 and 2000 is more than offset by the decrease in turbulent energy density, so that the total energy density decreases with increasing level of activity. Top: The ratio between radius change as a function of depth below the photosphere and radius change at $5 \mathrm{Mm}$ for model 4 . Note that the radius decreases with increasing solar activity.

the radius change as a function of radius to the radius change at $5 \mathrm{Mm}$.

From Figure 2 we note that in all cases the radius increases with increasing solar activity. This is to be expected since all the contribution of the magnetic field to pressure and internal energy is positive, and consequently, it can only lead to an increase of the radius. We also note that the increase of the radius is monotonic toward the surface. This is because the increase at a given radius is made up of the sum of the increase at all levels below it. Finally, we note that the expansion, which only increases in the magnetic region, is accelerated toward the shallower layers. This can be understood since, for a given value of the magnetic field, the ratio of magnetic to total pressure increases with increasing radius, and so does the expansion.

Figure 3 represents the case that, according to Li et al. (2003), meets all the observational requirements imposed by helioseismology. In particular, it produces the correct cycle-related variations of the $p$-mode oscillations, it does not alter the depth of the convection zone, and it produces diameter changes in opposite phase of the activity cycle. In this case, the magnetic field slows down turbulent flows so that the increase of magnetic pressure when the magnetic field grows is overcompensated by the corresponding decrease in turbulent pressure.
In all cases, the radius variation at the solar surface (which is measured by any limb-observing instrument, such as the Solar Disk Sextant [Sofia et al. 1994], and PICARD [Thuillier et al. 2005a]), can be hundreds of times larger than the radius variation inferred by $f$-mode oscillations, which represents changes at several megameters.

To determine the depth of the level of the "diameter" provided by the $f$-mode oscillations, we refer to Figure 1, which represents the kinetic energy of the modes of different $l$-values (abscissa in arbitrary units). We can see that for higher $l$-values, the peak energy occurs at shallower layers than for lower $l$ modes. It would appear that $5 \mathrm{Mm}$ is a good number to represent the depth of the layer given by $f$-mode oscillations of all degrees observed. Thus, the top panels of Figures 2 and 3 give the magnification factor between radius changes determined from $f$-mode oscillations, and the radius changes that can be expected at the photospheric level and thus observed by all limb-observing instruments.

Because $\Delta R$ increases in the shallower layers, the high $l$ modes, which peak in shallower layers, should show a larger radius change than the low $l$-modes, which peak at deeper layers. We believe that this is precisely what the results obtained by Antia \& Basu (2004) imply.

\section{SUMMARY AND CONCLUSIONS}

We have shown that the model of variability of the solar interior that obeys all observational constraints (global parameters and $p$-mode and $f$-mode oscillations) produces variations of the solar radius that increase by a factor of approximately 1000 from a depth of $5 \mathrm{Mm}$ to the solar surface. This model includes the effects of a variable dynamo magnetic field and of a field-modulated turbulence, and it explains features of the $f$-mode oscillations in different degree ranges that were previously not understood.

On the basis of the above argument, we conclude that the results from $f$-mode oscillations that the solar radius only changes by about $1 \mathrm{~km} \mathrm{yr}^{-1}$ do not preclude the less-sensitive efforts to measure variations of the solar radius at the photosphere by limb observations, since the latter are likely to be much larger than the former. Limb observations are made by a number of ground-based instruments, and from above the atmosphere by the Solar Disk Sextant (SDS) balloon-borne experiment (Sofia et al. 1994), and will be made starting in 2008 by the PICARD microsatellite (Thuillier et al. 2005a). In thermal equilibrium the space-based instruments have a theoretical precision of about 1 mas (about $1 \mathrm{~km}$ ). PICARD should easily reach such a precision. Balloon-based observations, however, cannot reach this precision because the short duration of the flights prevents the instrument from reaching thermal equilibrium. The current SDS results have reached a precision of the order \pm 0 ".05 (Egidi et al. 2005).

This work was supported in part by NSF grants ATM 0206130 and ATM 03-48837 to S. B.; S. S. and P. D. were supported in part by NASA grant NAG5-13299.

\section{REFERENCES}

Antia, H. M. 1998, A\&A, 330, 336

Antia, H. M., \& Basu, S. 2004, in Proc. SOHO 14/GONG 2004 Workshop:

Helio- and Asteroseismology: Toward a Golden Future, ed. D. Danesy (ESA SP-559; Noordwijk: ESA), 301
Antia, H. M., Basu, S., Pintar, J., \& Pohl, B. 2000, Sol. Phys., 192, 459 Antia, H. M., Basu, S., Pintar, J., \& Schou, J. 2001, in Proc. SOHO 10/GONG 2000 Workshop: Helio- and Asteroseismology at the Dawn of the Millennium, ed. A. Wilson (ESA SP-464; Noordwijk: ESA), 27 
Basu, S., Pinsonneault, M. H., \& Bahcall, J. N. 2000, ApJ, 529, 1084

Cox, J. P. 1980, Theory of Stellar Pulsation (Princeton: Princeton Univ. Press)

Dziembowski, W. A., Goode, P. R., di Mauro, M. P., Kosovichev, A. G., \& Schou, J. 1998, ApJ, 509, 456

Dziembowski, W. A., Goode, P. R., Kosovichev, A. G., \& Schou, J. 2000, ApJ, 537, 1026

Dziembowski, W. A., Goode, P. R., \& Schou, J. 2001, ApJ, 553, 897

Egidi, A., Caccin, B., Sofia, S., Twigg, L., Heaps, W., \& Hoegy, W. 2005, Sol. Phys., submitted

Li, L. H., Basu, S., Sofia, S., Demarque, P., \& Guenther, D. B. 2003, ApJ, 591, 1267

Li, L. H., Robinson, F. J., Demarque, P., Sofia, S., \& Guenther, D. B. 2002, ApJ, 567, 1192
Li, L. H., \& Sofia, S. 2001, ApJ, 549, L1204

Lydon, T. J., \& Sofia, S. 1995, ApJS, 101, 357

Robinson, F. J., Demarque, P., Li, L. H., Sofia, S., Kim, Y. C., Chan, K. L., \& Guenther, D. B. 2003, MNRAS, 340, 923

Schou, J., Kosovichev, A. G., Goode, P. R., \& Dziembowski, W. A. 1997, ApJ, 489, L197

Sofia, S., Heaps, W., \& Twigg, L. W. 1994, ApJ, 427, 1048

Thuillier, G., Dewitte, S., \& Schmutz, W. 2005a, Adv. Space Res., in press

Thuillier, G., Sofia, S., \& Haberreiter, M. 2005b, Adv. Space Res., 35, 329

Unno, W., Osaki, Y., Ando, H., Saio, H., \& Shibahashi, H. 1989, Nonradial Oscillations of Stars (2nd ed.; Tokyo: Univ. Tokyo Press)

Winnick, R. A., Demarque, P., Basu, S., \& Guenther, D. B. 2002, ApJ, 576, 1075 